\title{
Consistent realization of Celestial and Terrestrial Reference Frames
}

\author{
Younghee Kwak • Mathis Bloßfeld • Ralf Schmid • Detlef Angermann • \\ Michael Gerstl · Manuela Seitz
}

Received: date / Accepted: date

\begin{abstract}
The Celestial Reference System (CRS) is currently realized only by Very Long Baseline Interferometry (VLBI) because it is the space geodetic technique that enables observations in that frame. In contrast, the Terrestrial Reference System (TRS) is realized by means of the combination of four space geodetic techniques: Global Navigation Satellite System (GNSS), VLBI, Satellite Laser Ranging (SLR), and Doppler Orbitography and Radiopositioning Integrated by Satellite. The Earth orientation parameters (EOP) are the link between the two types of systems, CRS and TRS. The EOP series of the International Earth Rotation and Reference Systems Service were combined of specifically selected series from various analysis centers. Other EOP series were generated by a simultaneous estimation together with the TRF while the CRF was fixed. Those computation approaches entail inherent inconsistencies between TRF, EOP, and CRF, also because the input data sets are different. A combined normal equation (NEQ) system, which consists of all the parameters, i.e., TRF, EOP, and CRF, would overcome such an inconsistency. In this paper, we simultaneously estimate TRF, EOP, and CRF from an inter-technique combined NEQ using the latest GNSS, VLBI, and SLR data (2005-2015). The results show that the selection of local ties is most critical to the TRF. The combination of pole coordinates is beneficial for the CRF, whereas the combination of $\Delta \mathrm{UT} 1$ results in clear ro-
\end{abstract}

Younghee Kwak (汭 · Mathis Bloßfeld · Ralf Schmid · Detlef Angermann · Michael Gerstl · Manuela Seitz

Deutsches Geodätisches Forschungsinstitut at the

Technische Universität München (DGFI-TUM)

Arcisstr. 21, 80333 Munich, Germany

Tel.: +49-89-23031-1114

Fax: +49-89-23031-1240

E-mail: younghee.kwak@tum.de tations of the estimated CRF. However, the standard deviations of the EOP and the CRF improve by the inter-technique combination which indicates the benefits of a common estimation of all parameters. It became evident that the common determination of TRF, EOP, and CRF systematically influences future ICRF computations at the level of several $\mu$ as. Moreover, the CRF is influenced by up to $50 \mu$ as if the station coordinates and EOP are dominated by the satellite techniques.

Keywords Celestial Reference Frame - Terrestrial Reference Frame - ICRF · ITRF · Inter-technique combination $\cdot$ VLBI $\cdot$ SLR $\cdot$ GNSS $\cdot$ EOP

\section{Introduction}

In geodesy, there are two conventional reference frames, the International Celestial Reference Frame (ICRF) serving as quasi-inertial frame and the International Terrestrial Reference Frame (ITRF) which is fixed to the Earth's crust. Both frames are realizations of the theoretical (abstract) definitions of the International Celestial and Terrestrial Reference Systems (ICRS and ITRS; Arias et al., 1995). The transformation between ITRF and ICRF is represented by a time series of Earth orientation parameters (EOP).

ICRF solutions are created by a working group of the International Astronomical Union (IAU). The ICRS Centre of the International Earth Rotation and Reference Systems Service (IERS; Dick and Thaller, 2015), which is a joint service of the IAU and the International Union of Geodesy and Geophysics (IUGG), is in charge of their publication. Besides, the IERS is also responsible for the provision of ITRF solutions and their corresponding EOP. 
Nowadays, global TRF solutions are independently produced by three ITRS Combination Centres (CCs): the Institut National de l'Information Géographique et Forestière (IGN), the Deutsches Geodätisches Forschungsinstitut at the Technische Universität München (DGFI-TUM), and the Jet Propulsion Laboratory (JPL). Since 1989, the IGN computes ITRF solutions using a combination model based on Helmert transformation (Altamimi et al. 2002). DGFI-TUM obtains its TRF solutions (named DTRF) by the combination of normal equations (Seitz et al., 2012). The JPL TRF (named JTRF) is computed by employing the Kalman filter technique (Wu et al. 2015). The ITRF and DTRF solutions contain station positions and velocities of observing stations of different space geodetic techniques, namely the Global Navigation Satellite System (GNSS), Very Long Baseline Interferometry (VLBI), Satellite Laser Ranging (SLR), and Doppler Orbitography and Radiopositioning Integrated by Satellite (DORIS). The reference epochs are 2010.0 for the ITRF2014 (Altamimi et al. 2016) and 2005.0 for the DTRF2014 (Seitz et al., 2016). In contrast, JTRF consists of time series of station positions (Abbondanza et al., 2017). All three ITRS CCs use identical input data sets which are generated by IERS Technique Centres: the International GNSS Service (IGS; Dow et al., 2009), the International VLBI Service for Geodesy and Astrometry (IVS; Schuh and Behrend, 2012), the International Laser Ranging Service (ILRS; Pearlman et al., 2002), and the International DORIS Service (IDS; Willis et al., 2010). However, different combination strategies applied by the ITRS CCs (and institute-specific data editing steps) result in discrepancies between the TRF solutions. Bloßfeld et al. (2017) address the distinct scale differences between the latest TRF solutions. At the same time, the cross-validation of these realizations allows for a quantification of the current accuracy of the ITRF product.

The latest ICRF at radio wavelengths is the ICRF2 (Fey et al. 2015) which consists of positions of compact astronomical radio objects and was adopted by the IAU in January 2010. The ICRF2 radio source positions are based on nearly 30 years of VLBI observations which are coordinated by the IVS since 1999 .

The 3414 ICRF2 radio sources can be split into two major groups. About two thirds (2197) of all sources were only observed by the Very Long Baseline Array (VLBA; Petrov et al. 2009) within the scope of the socalled VLBA Calibrator Surveys (VCS). Between 1994 and 2007, six multi-session VCS campaigns were held comprising a total of 24 separate 24 -h sessions (e.g., Beasley et al., 2002, Petrov et al., 2008). As most of these "VCS-only" sources were only observed in one session (Fey et al. 2015), their precision is about five times worse compared to "non-VCS" sources (Gordon, 2014).

Among the remaining third of 1217 non-VCS sources are 295 "defining" sources designated to define the axes (orientation) of the ICRF2. To achieve a uniform sky distribution, the most stable and most frequently observed sources with minimum intrinsic source structure were selected from certain declination bands. The 39 sources exhibiting the largest position variations are called "special handling" sources. To avoid the distortion of multi-year reference frames, their positions should either be treated as session-wise parameters or reduced. Meanwhile, it is worth mentioning here that not only the special handling sources, which were selected 8 years ago, but also the other sources including defining sources suffer from strong structure effects. Therefore, additional time-series analysis of the sources should be studied for a proper treatment of them.

The preparation of the next-generation ICRF is already in progress (Malkin et al., 2015). Besides the extension to higher-frequency bands and the preparation of the link to a Gaia-based optical frame, a substantial improvement in the legacy S/X-band is a major goal of the ICRF3 effort. Since over recent years several new VLBI antennas were installed in the southern hemisphere, especially in Australia (Lovell et al., 2013), the ICRF is expected to benefit both in terms of a uniform distribution and the position accuracy of its southern sources.

To reduce the position uncertainty of the VCS-only sources, a new VCS campaign was initiated (Gordon, 2014). VCS-II comprised eight 24-h sessions that were observed between January 2014 and March 2015. With more than 300 sources per session observed at $2 \mathrm{Gbit} / \mathrm{s}$, it was possible to re-observe all VCS-only together with many new sources. Due to improvements in the VLBA data acquisition, the source positions could be significantly improved by VCS-II (Malkin et al., 2015; Gordon et al. 2016).

The EOP connecting an ITRF and an ICRF are composed of five parameters. The two celestial pole offsets provide corrections to a conventional precessionnutation model, two parameters describe the polar motion w.r.t. the Earth's crust, and one gives the irregularities of its angular velocity. The latter is defined by (see also Bloßfeld, 2015)

$$
\begin{aligned}
& \Delta \mathrm{UT} 1:= \\
& \quad=\mathrm{UT} 1-\mathrm{UTC}-\Delta \mathrm{UT} 1_{\text {ocean tides }}-\Delta \mathrm{UT} 1_{\text {libration }} \\
& \quad=(\text { "UT1-UTC") })_{\text {IERS }}
\end{aligned}
$$

and thus, it is directly comparable with the so-called UT1-UTC parameter of the IERS 14 C04 time series 


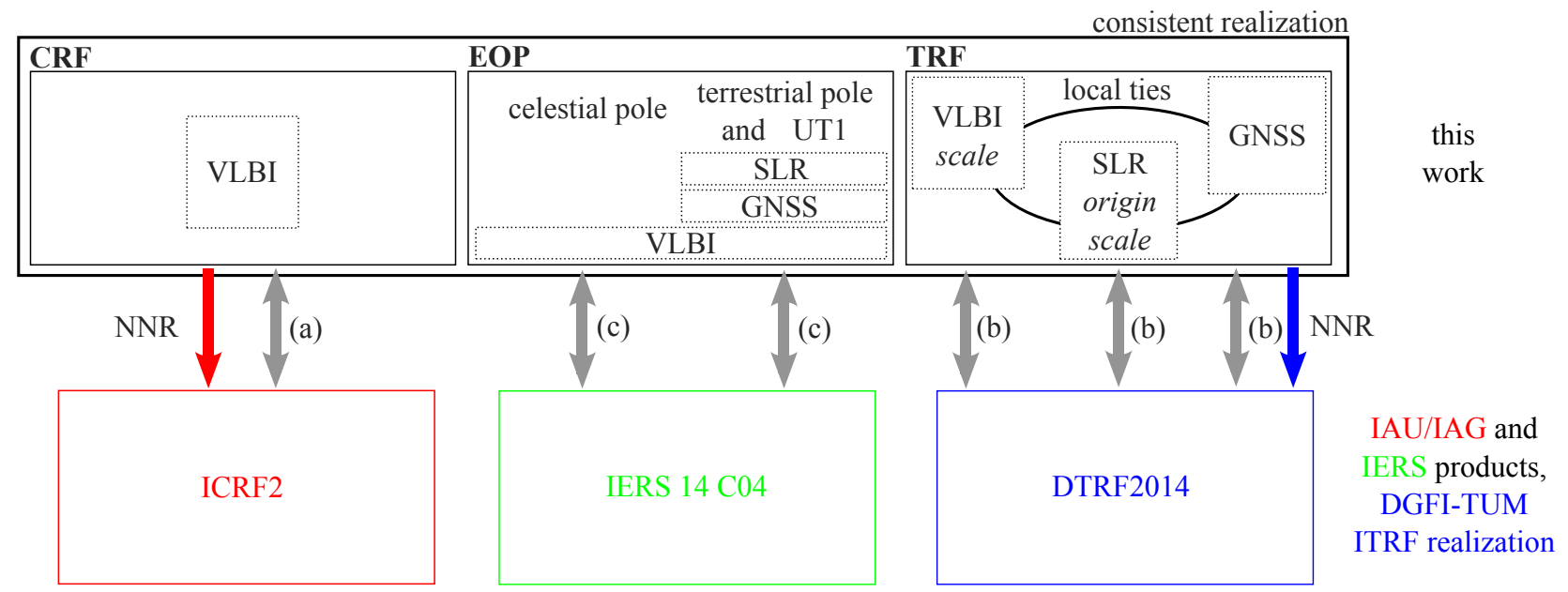

Fig. 1 Consistent realization of CRF, TRF, and EOP as computed in this investigation. The official IAU/IAG and IERS products and the DGFI-TUM ITRF realization are used as a priori values for each parameter group to realize a stable and reliable datum realization and for validation purposes. The CRF solutions are compared by a CRF transformation (a) using six parameters shown in Eq. 2]. The TRF solutions are compared using a TRF transformation (b) with $7 / 14$ parameters. In case of the EOP, differences of the time series are analyzed (c).

(Bizouard and Gambis, 2017). The excess length-ofday $(\Delta \mathrm{LOD})$, which is related to the time derivative of $\Delta \mathrm{UT} 1$, is defined by (see also Bloßfeld, 2015)

$$
\Delta \mathrm{LOD}:=-86400 \mathrm{~s} \frac{d}{d t} \Delta \mathrm{UT} 1
$$

This parameter is given under the column header "LOD" in the IERS C04 series. The reference time series for our EOP solution is IERS $14 \mathrm{C} 04$. It is the result of a combination of operational EOP time series provided by different space geodetic techniques (Bizouard and Gambis, 2017) and commonly used as reference in the geodetic community.

Within Resolution 3 adopted by the General Assembly in 2011, the IUGG (2011) urged "that highest consistency between the ICRF, the ITRF, and the EOP as observed and realized by the International Association of Geodesy (IAG) and its components such as the IERS should be a primary goal in all future realizations of the ICRS." However, the highest consistency could only be achieved, if all three components are estimated simultaneously in one common adjustment (e.g., Seitz et al. 2014). Up to now, three different IERS Product Centres are in charge of the two reference frames and the EOP, namely the IERS ICRS Centre, the IERS ITRS Centre, and the IERS Earth Orientation Centre (Dick and Thaller, 2015).

To derive a C04 solution, the IERS Earth Orientation Centre tries to achieve consistency with ICRF and ITRF by aligning all operational series with some selected series that are assumed to be consistent with ICRF and ITRF (Bizouard and Gambis, 2017). In the case of the ICRF2, one argument to prefer the solution of a single analysis center to a combined solution of multiple analysis centers was the assumption that a single catalog could be more consistent with ITRF2008 and the official EOP (Fey et al., 2015). In contrast, the ICRF2 was not generated consistently with the final ITRF2008 but with the IVS input to that ITRS realization, called VTRF2008 (Böckmann et al., 2010). However, the two frames are not fully consistent, as the VTRF scale is realized from VLBI only, whereas the ITRF2008 scale is a weighted mean of VLBI and SLR (Seitz et al., 2014). In addition, the origin of the VTRF is realized by a no-net-translation (NNT) condition over a selected subnet of VLBI positions, whereas the ITRF origin is realized by SLR. Moreover, the network geometries differ due to the effect of combination on the station coordinates which is caused by the introduction of terrestrially measured difference vectors between reference points (local ties; LTs) and the equating of station velocities.

In this paper, we simultaneously estimate CRF, TRF, and the linking EOP series using 11-year VLBI, SLR, and GNSS data to reach consistency between CRF and TRF (see Fig. 1). In Sect. 2, the input data of the epoch-wise single-technique solutions are presented. Section 3 discusses the time-series analysis performed with the single-technique epoch-wise solution to ensure suitable normal equations (NEQs) for the rigorous combination. Afterward, Sect. 4 describes the combination strategy applied at DGFI-TUM to compute a multi-year multi-technique solution which contains the above-mentioned parameters. Using this solution, 
Table 1 Setups of the multi-year single-technique NEQ systems used for the combination in this investigation. The datum conditions are applied in order to obtain multi-year single-technique solutions for validation purposes.

\begin{tabular}{|c|c|c|c|}
\hline & VLBI & SLR & GNSS \\
\hline Institution & DGFI-TUM & DGFI-TUM & $\mathrm{CODE}^{a}$ \\
\hline \multirow[t]{2}{*}{ Software } & OCCAM (Titov et al. 2004) & DOGS-OC & Bernese GNSS Software \\
\hline & & (Gerstl, 1997 Bloßfeld, 2015) & (Dach et al. 2015) \\
\hline Resolution & Session-wise & Weekly & Daily \\
\hline Stations reduced? & $\begin{array}{l}\text { Yes, if observations in less than } \\
10 \text { sessions are given }\end{array}$ & $\begin{array}{l}\text { Yes, if less than } 10 \text { normal } \\
\text { points per arc or less than } 10 \\
\text { weeks within the time interval }\end{array}$ & No special handling known \\
\hline Datum conditions & $\begin{array}{l}\mathrm{NNR}^{b} / \mathrm{NNT}^{c} \text { to DTRF2014, } \\
\text { NNR to ICRF2 }\end{array}$ & NNR to DTRF2014 & NNR/NNT/NNS ${ }^{d}$ to DTRF2014 \\
\hline
\end{tabular}

${ }_{a}^{a}$ The Center for Orbit Determination in Europe (Steigenberger et al., 2014)

${ }^{b}$ no-net-rotation, ${ }^{c}$ no-net-translation, ${ }^{d}$ no-net-scale

we assess the impact of various LT selections, different EOP combination setups, and the down-weighted VLBI NEQ on the CRF, TRF, and EOP. The benefits and limitations of the presented combination results are discussed in the last section. The outcome of this investigation should be a statement or a quantification whether, and in what way, consistently estimated CRF, TRF, and EOP are beneficial for different parameter groups. This investigation also supports the ambitious goal of the Global Geodetic Observing System (GGOS; Rothacher et al., 2009) to consistently estimate fundamental geodetic parameters (or products) from multiple space geodetic techniques.

\section{VLBI, SLR, and GNSS input data}

In this study, we focus on the combination of homogeneously processed VLBI, SLR, and GNSS data on the normal equation level to simultaneously estimate CRF, TRF, and the linking EOP. We consider this as an exemplary study by also using observation data over a time period of 11 years only to investigate the impact of different solution setups. A full combination with all four space geodetic techniques (including DORIS) is planned for the future.

In case of VLBI and SLR, we reprocessed the input data to be consistent with officially available GNSS products derived by the Center for Orbit Determination in Europe (CODE). Details of the different techniquespecific NEQs can be found in Table1. The included unknowns are listed in Table 2. Each NEQ covers exactly the same time span (2005.0-2016.0) so that the contribution of the single techniques could be regarded as homogeneous w.r.t. the time interval. A datum test was performed to ensure that only datum-free NEQs are included in the combination process. Details on this check are given in Bloßfeld (2015). Moreover, discontinuities according to the DTRF2014 processing are introduced (Seitz et al., 2016) to account for, among others, earthquakes (impact on station coordinate and/or velocity) and instrument changes. For all NEQ systems, the official products of IAU/IAG (ICRF2), IERS (14 C04 time series) as well as the conventional realization of DGFITUM (DTRF2014 with station coordinates and velocities) are used as a priori values to ensure a stable and reliable datum realization and for validation purposes.

The VLBI data were processed using the OCCAM software (Titov et al. 2004). The estimated parameters from VLBI data were station coordinates, sources coordinates, EOP, troposphere and clock parameters during session-wise estimation. Then, the tropospheric and clock parameters were reduced, and datum-free NEQs that contain station coordinates, source coordinates, and EOP are generated for the multi-year solutions.

In case of SLR, the "Orbit Computation" (OC) library of the "DGFI Orbit and Geodetic parameter estimation Software" (DOGS-OC; Gerstl, 1997. Bloßfeld, 2015) was used to process the SLR data. A satellite-specific observation outlier detection was performed within the iterative precise orbit determination (POD) of the two used spherical satellites LAGEOS1 and LAGEOS-2. The orbit length within this POD process is 7 days over the whole time span. The resulting NEQs contain only station coordinates and EOP since satellite-specific orbit parameters, empirical accelerations, and scaling factors for selected nonconservative perturbing forces are reduced. It has to be mentioned here that the $\frac{d}{d t}(\Delta \mathrm{UT} 1)$ estimates of the satellite techniques are corrupted by spurious signals since $\frac{d}{d t}(\Delta \mathrm{UT} 1)$ is highly correlated with orbit parameters and the low degree spherical harmonics of the Earth's gravitational field (Bloßfeld, 2015). All EOP are parameterized as piece-wise linear polygons. For the satellite techniques, this parameterization is also chosen 
Table 2 Characteristics of the single-technique epoch-wise (no station velocities included in NEQ) and multi-year solutions. The origin is realized in the SLR-only solutions, and scale is realized in the SLR-only and VLBI-only solutions intrinsically. The NNT, NNR, and NNS conditions for the TRF are applied to a technique-specific subnet of stable station coordinates only. The NNR condition for the CRF is applied to the defining sources only.

\begin{tabular}{|c|c|c|c|c|}
\hline & & VLBI & SLR & GNSS \\
\hline \multirow{5}{*}{ 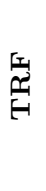 } & Station coordinates & $\mathrm{X}$ & $\mathrm{X}$ & $\mathrm{X}$ \\
\hline & Station velocities & $\mathrm{X}$ & $\mathrm{X}$ & $\mathrm{X}$ \\
\hline & Origin & NNT & intrinsic & NNT \\
\hline & Scale & intrinsic & intrinsic & NNS \\
\hline & Orientation & NNR & NNR & NNR \\
\hline \multirow{2}{*}{ 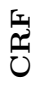 } & Source coordinates & $\mathrm{X}$ & & \\
\hline & Orientation & NNR & & \\
\hline \multirow{3}{*}{$\underset{|c|}{0}$} & Terrestrial $x / y$-pole & $\mathrm{X}$ & $\mathrm{X}$ & $\mathrm{X}$ \\
\hline & Celestial $X / Y$-pole & $\mathrm{X}$ & & \\
\hline & $\Delta \mathrm{UT} 1 *$ & $\mathrm{X}$ & $(\mathrm{X})$ & $(\mathrm{X})$ \\
\hline
\end{tabular}

$\left(^{*}\right)$ for the satellite techniques, one $\Delta \mathrm{UT} 1$ value per solution is fixed to a priori

for $\Delta \mathrm{UT} 1$ even if these techniques are not able to determine this parameter absolutely, but only $\frac{d}{d t}(\Delta \mathrm{UT} 1)$. The information of $\frac{d}{d t}(\Delta \mathrm{UT} 1)$ is included in the piecewise linear $\Delta \mathrm{UT} 1$ polygons indirectly. This type of parameterization provides a higher homogeneity of the estimated parameters w.r.t. precision than the parameterization with an offset $\Delta \mathrm{UT} 1$ and a drift $\frac{d}{d t}(\Delta \mathrm{UT} 1)$ at the mean epoch of the day (GNSS), session (VLBI), or week (SLR).

The GNSS NEQs were obtained from the Center for Orbit Determination in Europe (CODE; Steigenberger et al., 2014). Thereby, the NEQs between 2005.0 and 2015.0 were generated in the framework of the reprocessing campaign of the IGS (CODE is an IGS Analysis Center) which were used for the combined IGS input solution for the ITRF2014. The NEQs between 2015.0 and 2016.0 were generated routinely at CODE with comparable a priori models. All daily GNSS NEQs contain station coordinates and two offsets at the midnight epochs for each EOP.

\section{Time series analysis}

For a reliable combination result, it is crucial to ensure that the single-technique multi-year NEQs are suitable for a rigorous combination. A special focus must be put on the datum definition and degrees of freedom in each NEQ to avoid over-constraining and potential deformations of networks. To ensure a stable and reliable velocity estimation, all epoch-wise technique- specific NEQs are solved (specific datum conditions applied; see Table 2. In case of the satellite techniques, at least one $\Delta \mathrm{UT} 1$ parameter has to be fixed to its a priori value. The resulting time series of station coordinates are checked for outliers ( $3 \sigma$-criterion) which are reduced from the original NEQs. In addition, the epochwise datum parameters of each technique-specific NEQ are evaluated. The origin and the scale are physical datum parameters, which means that they are intrinsically realized by SLR (origin, scale) and VLBI (scale). Figure 2 shows the estimated epoch-wise translation offsets of the SLR-only solutions w.r.t. DTRF2014 (upper three panels) and the estimated scale offsets of the VLBI-only and SLR-only solutions w.r.t. DTRF2014 (lowest panel). For the TRF transformation as well as for the applied datum conditions shown in Table 2, only stable stations with a long observation interval were used (selection based on outlier detection described before). The analysis of these time series allows to validate the intrinsically realized TRF datum of both singletechnique solutions.
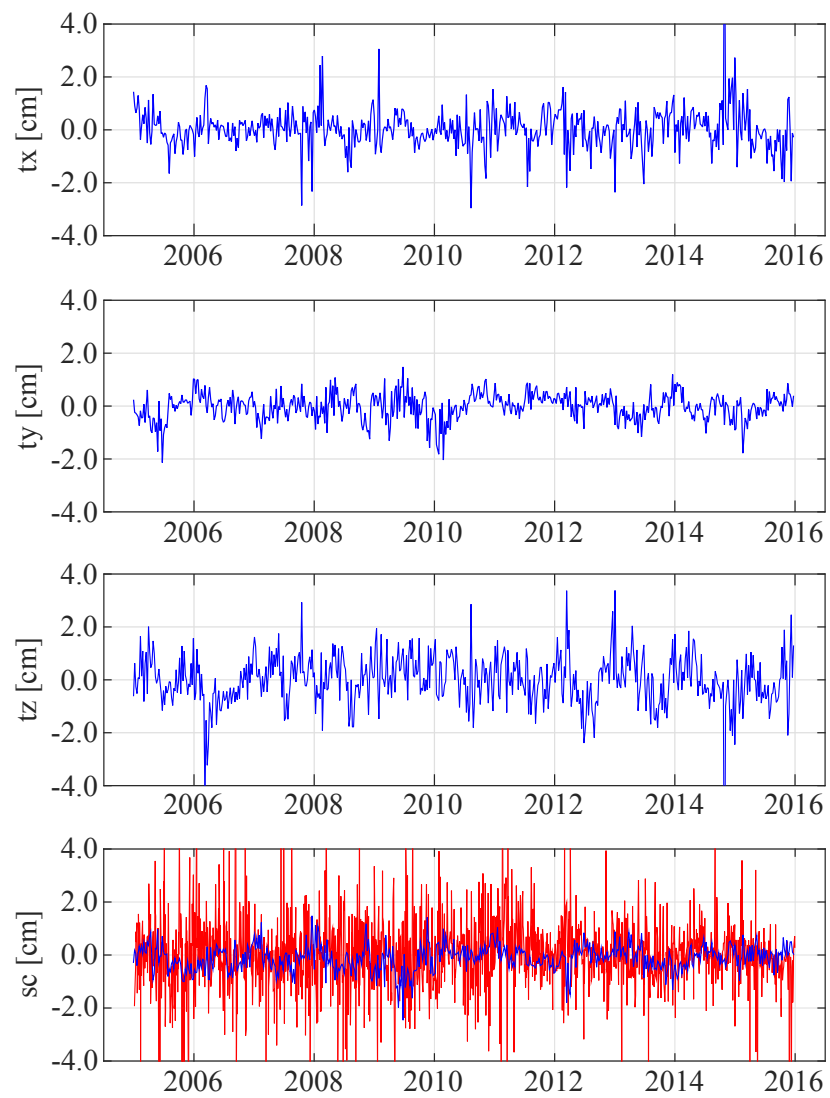

Fig. 2 Time series of epoch-wise estimated translation and scale offsets of the weekly/session-wise SLR-/VLBI-only (blue/red) solutions w.r.t. DTRF2014. 
For the translation time series, the scatter in all components is, in general, clearly below $\pm 2.0 \mathrm{~cm}$ which proves that the weekly SLR-only solutions realize a stable origin throughout the whole time interval. As both techniques contribute to the scale of the combined reference frame, the consistency between them is a prerequisite. The scatter of the VLBI-only scale offsets shown in Fig. 2 is larger than the scatter of the SLR-only scale offsets due to alternating VLBI session types with varying VLBI observation stations and a patchy daily resolution. Thereby, a regional VLBI session realizes a scale that suffers from a poor network geometry. Nevertheless, a systematic bias between both time series could not be clearly identified which means that the realized SLR-only and VLBI-only scales agree well to each other. The estimated EOP and the CRF parameters were compared to the IERS 14 C04 time series and the ICRF2, but no systematic offsets were found.

\section{Combination strategy}

In this section, the combination strategy applied at DGFI-TUM is presented. Basically, it can be divided into two major steps: (i) the intra-technique combination and (ii) the inter-technique combination.

The intra-technique combination accumulates all epoch-wise NEQs of a single technique to one big technique-wise multi-year NEQ. At the outset of that combination, station velocities are introduced as additional parameters with a priori values derived from the DTRF2014. If discontinuities are introduced for a station, the velocities of such interval-solutions are regarded as being identical if they are nearly equal. This decision was based on a defined threshold listed in detail in Table 3. Afterward, all the station coordinates are transformed to a common epoch (e.g., 2011.0). Within the NEQ accumulation, the positions and velocities are equalized per station. Stations with too less observations (less than 10 epochs) or a too short observation time span (less than 2.5 years) are reduced to avoid instabilities of the accumulated NEQ.

Since the source positions are generally considered as constant in time, the variables of the source coordinate corrections of different sessions are set equal when adding NEQs. The only exception are the 39 special handling sources which are not equated but treated as time series. All EOP are equalized at the day/session/week boundaries which results in only one value per day for each component. The stacking of $\Delta \mathrm{UT1}$ values at midnight epochs is possible since these unknowns are parameterized as piece-wise linear functions which include the $\frac{d}{d t}(\Delta \mathrm{UT} 1)$ parameters indirectly. In total, the multi-year VLBI-only NEQ con- tains 69 stations and 3518 sources including 284 defining and 39 special handling sources. The SLR-only multi-satellite NEQ contains 56 stations and 4026 daily parameters for each of the $x / y$-pole coordinates and $\triangle \mathrm{UT} 1$ offsets. Concerning GNSS, 4025 daily SINEX files were accumulated to one GNSS-only multi-year NEQ which comprises 658 stations and 4026 daily EOP.

Solving for the individual satellite techniques as described for the epoch-wise case in Sect. 2 and estimating the EOP, at least one $\Delta \mathrm{UT} 1$ parameter has to be fixed to its a priori value. In the case of the combined solution, this constraint is not necessary since VLBI supports the satellite technique relative $\Delta \mathrm{UT} 1$ information with absolute values. A major question within this study is to what extent the (systematically affected) satellite technique $\Delta \mathrm{UT} 1$ values impact the absolutely determined VLBI $\Delta \mathrm{UT} 1$ values. The TRF datum of the single-technique multi-year solutions is realized as described in Table 2 in principal in the same way as for the epoch-wise technique-specific solutions except the fact that the TRF datum conditions are extended from the 7-parameter to 14-parameter conditions (also the rates of the TRF datum parameters are constrained).

In the second step, the inter-technique combination, the technique-specific multi-year NEQs are relatively weighted, stacked and additional constraints and conditions are applied. The constraints for the combination of station networks comprise terrestrial difference vectors between station reference points (local ties) and velocity constraints to equalize statistically similar velocities. The conditions for the datum realization are an NNR condition w.r.t. a selected subnet of stable and globally homogeneous distributed GNSS stations and an NNR condition w.r.t. the defining sources of the ICRF2. The EOP are directly stacked since they are common to all techniques (see Table 2). Afterward, the combined NEQ is inverted using the "Combination and Solution" (CS) library of DOGS (Gerstl et al., 2000 Angermann et al., 2004).

As a result of this processing strategy, we obtain the following solutions which can be inter-compared to each other and to external solutions:

- technique-specific epoch-wise solutions; these solutions are used to perform time-series analysis as described in Sect. 2,

- technique-specific multi-year solutions; these solutions are used to quantify the stability of the geodetic datum as well as the deformation of the techniquespecific station networks due to the inter-technique combination,

- inter-technique multi-year solution; this solution contains the consistently estimated TRF, EOP, and CRF parameters. 


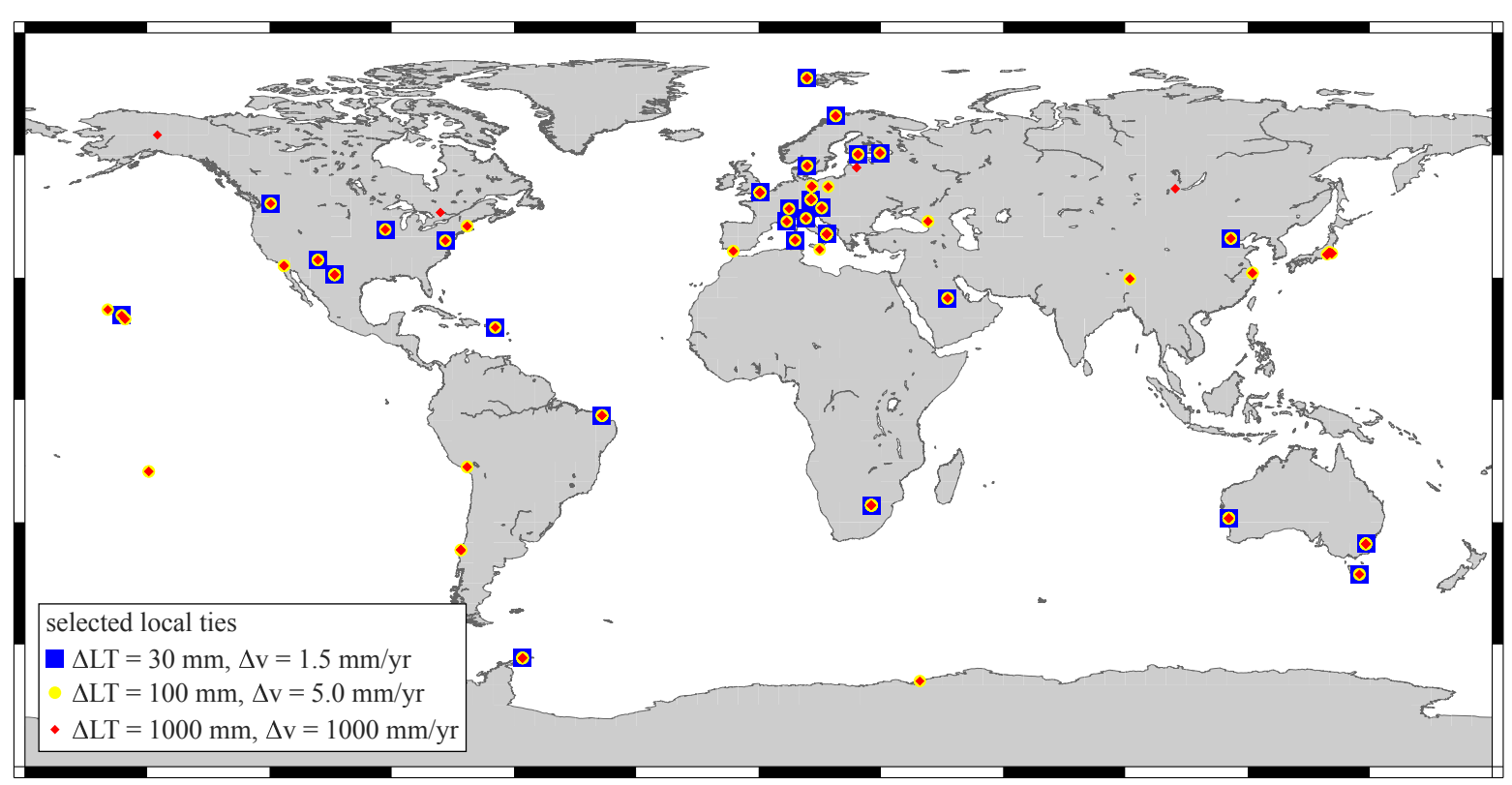

Fig. 3 Selected co-located sites according to different local tie thresholds described in Table 3

Table 3 Number of introduced LTs in solution types according to different $\mathrm{LT}$ and velocity equality threshold ( $\triangle L T$ and $\Delta v)$ selections. Solution (A), where all EOP are combined and where the weights between techniques are identical, is the reference solution (see also Table 4 ).

\begin{tabular}{lccc}
\hline Solution & $(\mathrm{A})$ & $(\mathrm{B})$ & $(\mathrm{C})$ \\
& $\begin{array}{c}\Delta \mathrm{LT}< \\
\Delta \mathrm{v}<1.5 \frac{\mathrm{mm}}{\mathrm{yr}}\end{array}$ & $\begin{array}{l}100 \mathrm{~mm} \\
5 \frac{\mathrm{mm}}{\mathrm{yr}}\end{array}$ & $1000 \mathrm{~mm}$ \\
& 32 & 44 & 47 \\
\hline GNSS/GNSS & - & 1 & 4 \\
VLBI/VLBI & 23 & 55 & 135 \\
GNSS/VLBI & 30 & 60 & 82 \\
GNSS/SLR & 4 & 7 & 14 \\
SLR/VLBI & 89 & 167 & 282 \\
Total & & & \\
\hline
\end{tabular}

In the following, we focus on the inter-technique multi-year solution and evaluate different solution setups to study the interaction mechanisms between the different parameter groups (see Table 4 ).

\subsection{Selections of Local Tie and Velocity Equality}

Since the coordinates of technique-specific reference points are no common parameters to all techniques, terrestrially measured difference vectors, so -called LTs, are necessary to combine the reference points on ground. The LTs are absolutely crucial elements for the inter-technique combination and the realization of a common TRF datum to all technique-specific subnets in the combined frame (Angermann et al., 2013; Seitz et al. 2012). The ITRS Center provides all LT SINEX files ${ }^{1}$ used, e.g., in the ITRF2014 combination. In addition, the velocities of the reference points at co-located sites are assumed to be equal because one would expect that both points are influenced by the same geophysical phenomena. Both types of constraints, the LTs and the velocity constraints, are introduced into the NEQ system as pseudo-observation equations. The selection of

1 http://itrf.ensg.ign.fr/local_surveys.php

Table 4 Solution setups to investigate different impacts on a consistent estimation of CRF, TRF, and EOP. The highlighted setups are compared to solution (A). Details on the comparisons are given in Sect. 4. the results are discussed in Sect. 5

\begin{tabular}{cccc}
\hline solution & $\begin{array}{c}\Delta \mathrm{LT}[\mathrm{mm}] / \\
\Delta v[\mathrm{~mm} / \mathrm{yr}]\end{array}$ & $\begin{array}{c}\text { Which EOP } \\
\text { are combined? }\end{array}$ & $\begin{array}{c}\text { Weighting } \\
\text { of } \\
\text { techniques }\end{array}$ \\
\hline (A) & $30 / 1.5$ & All & Equal weights \\
(B) & $100 / 5.0$ & All & Equal weights \\
(C) & $1000 / 1000$ & All & Equal weights \\
\hline (D) & $30 / 1.5$ & $\begin{array}{c}\text { VLBI-only, } \\
\text { SLR/GNSS }\end{array}$ & Equal weights \\
(E) & $30 / 1.5$ & $\Delta \mathrm{UT1}$ only & Equal weights \\
(F) & $30 / 1.5$ & $x / y$-pole only & Equal weights \\
\hline (G) & $30 / 1.5$ & All & $\begin{array}{l}\text { VLBI NEQ: } \\
\lambda_{\text {VLBI }}=0.1\end{array}$ \\
\hline
\end{tabular}


the LT and velocity constraints rely on statistical tests. For the LTs, the single-technique multi-year solutions are used to compute the reference point difference vector at the measurement epoch of the LT. The points are interpolated to this epoch $t_{\mathrm{LT}}$ using the positions at the reference epoch of the TRF and the consistently estimated velocities. The decision if the LT is introduced is made according to the threshold

$$
\Delta \mathrm{LT}=\left\|\mathbf{L T}\left(t_{\mathrm{LT}}\right)-\left(\boldsymbol{X}_{1}\left(t_{\mathrm{LT}}\right)-\boldsymbol{X}_{2}\left(t_{\mathrm{LT}}\right)\right)\right\|_{2}
$$

The 3D-discrepancy $\Delta \mathrm{LT}$ could be caused by various geophysical, technical, or anthropogenic effects such as, e.g., earthquakes, antenna changes, systematic effects of the antenna reference points, or measurement errors. In Eq. (1), $\boldsymbol{X}_{1}$ and $\boldsymbol{X}_{2}$ are the 3D-coordinates of the single-technique multi-year solution of techniques 1 and 2. If $\Delta \mathrm{LT}$ is smaller than a defined value, the LT is introduced. As thresholds, three different values of 30,100 , and $1000 \mathrm{~mm}$ are selected. These values have been chosen since they categorize significant changes in the number of LTs, especially between SLR and VLBI which is the crucial constraint for the quality of the TRF scale realization. The global coverage and the exact numbers of LTs depending on the different thresholds are shown in Fig. 3 and Table 3 .

The weighting of the LT constraints is done according to the $3 \mathrm{D}$-discrepancy $\Delta \mathrm{LT}$. The weight used in the combination process is $\lambda=(5 / \Delta \mathrm{LT})^{2}$ with $\Delta \mathrm{LT}$ (in $\mathrm{mm})$. As it is the case for the $\Delta \mathrm{LT}$ values, also the velocity constraints are selected w.r.t. a certain threshold. It is varied together with the $\Delta \mathrm{LT}$ constraints as shown in Table 3. The solution which is indicated by $(\mathrm{A})$ in Table 3 serves as the reference solution in this investigation since we know from studies within the DTRF2014 processing that these thresholds do not cause a significant deformation of the single-technique subnets in the combined frame. In case of the ITRF2014 and JTRF2014, no a priori LT selection is performed. Both TRF solutions use all available LTs and introduce them using relative weights according to their 3 -D fit w.r.t. the single-technique solution differences.

\subsection{EOP combination setups}

The EOP are common parameters to all space geodetic techniques. GNSS provides daily terrestrial pole coordinates with a good precision because of the homogeneous global distribution of stations and its continuous observations. As a result, GNSS is dominant in the determination of terrestrial pole coordinates among the space geodetic techniques. Those precise terrestrial pole coordinate series are expected to improve the link between the TRF and CRF. The combination with satellite techniques would also be advantageous in respect of continuity at day boundaries because the regular VLBI observation campaigns (R1 and R4 sessions) are usually held only once per week, each. The IVS Intensive sessions are not considered here because their parameterization is different from the regular sessions. According to Seitz et al. (2014), the combination of the EOP can have a systematic effect on the estimated source positions which are observed by regional station networks with few observations only. In order to check the impact of combined EOP on the CRF as well as on the TRF in more detail, four different setups of EOP combinations are investigated in this study (see Table 4). Solution (A) serves again the reference solution. In solution (D), the VLBI EOP are not combined at all to the satellite technique derived EOP. In the solutions (E) and (F), $\triangle \mathrm{UT} 1$ or the terrestrial pole coordinates are combined, respectively.

\subsection{Weighting of techniques}

All multi-year single-technique solutions are re-scaled according to their a posteriori variance factors $\hat{\sigma}_{0}^{2}$ before the combination. This is done in order to ensure that all NEQs to be combined have a comparable variance level. For GNSS, it is well known that the estimated standard deviations are too optimistic due to the not considered high correlations of the GNSS observations. This deficiency cannot be considered by inter-technique weighting, and the high GNSS precision is also present within the combined solution. Afterward, in the combination, the re-scaled NEQs are all equally weighted. As a last test scenario, we down-weight the VLBI NEQ in the combination by a factor of $\lambda_{\mathrm{VLBI}}=0.1$ to test the impact on the parameters when the combination is dominated by satellite techniques (see Table 4 ).

\section{Results}

This section summarizes the results obtained from the investigation of different combination scenarios on consistently estimated TRF, EOP, and CRF parameters. In total, 7 different combination setups are investigated which are summarized in Table 4. The comparisons of the different parameter groups are done using a 6parameter CRF transformation and a 14-parameter TRF transformation, and analyzing EOP difference time series.

The CRFs are compared with each other by means of the CRF transformation model (Fey et al., 2009, har- 
monic terms are ignored):

$\Delta \alpha=\left(A_{1} \cos \alpha+A_{2} \sin \alpha\right) \tan \delta-A_{3}+D_{\alpha}\left(\delta-\delta_{0}\right)$,

$\Delta \delta=-A_{1} \sin \alpha+A_{2} \cos \alpha+D_{\delta}\left(\delta-\delta_{0}\right)+B_{\delta}$.

Here, $\alpha$ and $\delta$ represent the right ascension and declination of source coordinates and $\Delta \alpha$ and $\Delta \delta$ mean the differences of them between two CRFs. $A_{1}, A_{2}$, and $A_{3}$ denote the rotation between two CRFs w.r.t. the three axes. $D_{\alpha}$ and $D_{\delta}$ represent the drifts of right ascension and declination w.r.t. a reference declination $\delta_{0}\left(\delta_{0}\right.$ is usually $0 \mathrm{deg}) . B_{\delta}$ means the bias in declination. In the following, this transformation will be denoted as CRF transformation with CRF transformation parameters.

It has to be mentioned here that these transformation parameters are correlated to each other. For example, $A_{2}$ is correlated to $D_{\delta}$ and $B_{\delta}$ by about -0.6 ( $A_{1}$ by about -0.3$)$ whereas $A_{3}$ is correlated to $D_{\alpha}$ by about 0.8 . This means, systematic effects in the declinations of the source positions impact the parameters $A_{1}, A_{2}$, $D_{\delta}$, and $B_{\delta}$, and the rotation $A_{3}$ can be associated with a systematic effect in the right ascension $\left(D_{\alpha}\right)$.

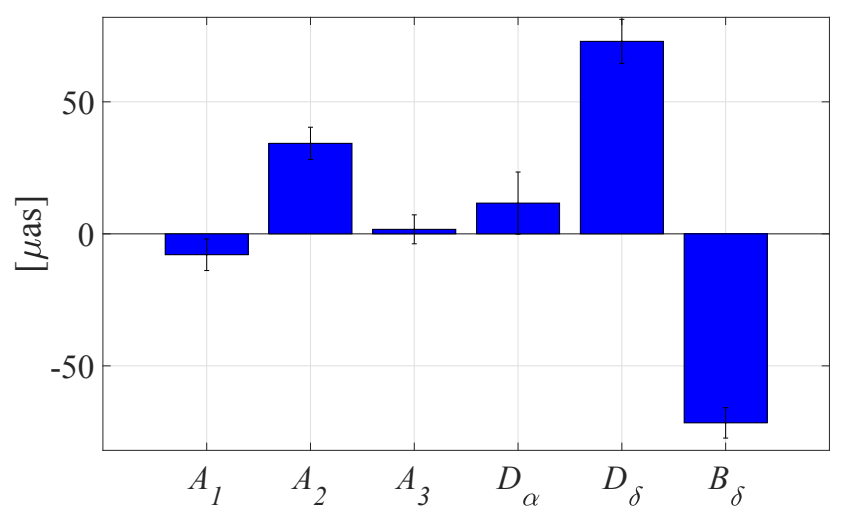

Fig. $4 \mathrm{CRF}$ transformation parameters and their standard deviations (error bars) of VLBI-only w.r.t. ICRF2.

Figure 4 shows the CRF transformation parameters of Eq. (2) of the VLBI-only w.r.t. ICRF2 using only defining sources. It should be noted that ICRF2 is based on VLBI observations between 1979 and 2009 while the solutions of this study cover the data period 2005-2015. As is well known in the VLBI community, the inclusion of Australian stations since 2010 has caused biases $\left(B_{\delta} \approx 70-80 \mu \mathrm{as}\right)$ in the declination of the CRF w.r.t. the ICRF2. Since the data period of this study includes the most recent years (and also the observations of the Australian stations), those biases could also be detected clearly in the VLBI-only (Fig. 4) and also in the combined solutions (not directly shown). This fact also explains the estimated rotations w.r.t. ICRF2 although an NNR condition w.r.t. ICRF2 was applied. If only the three rotations $A_{1}, A_{2}$, and $A_{3}$ are estimated, only an offset in $A_{2}$ is significantly estimated which is caused by the systematic declination bias between ICRF2 and our CRF solution.

Despite this bias, the VLBI-only solution matches well with the ICRF2, especially in the $A_{3}$ component (Fig. 4), which is related to the Earth rotation, because $\triangle \mathrm{UT1}$ is solely based on VLBI data both in the VLBIonly CRF and in the ICRF2. In the following sections, the CRF comparison between VLBI-only and combined solutions will be presented.

\subsection{Impact of LT selections}

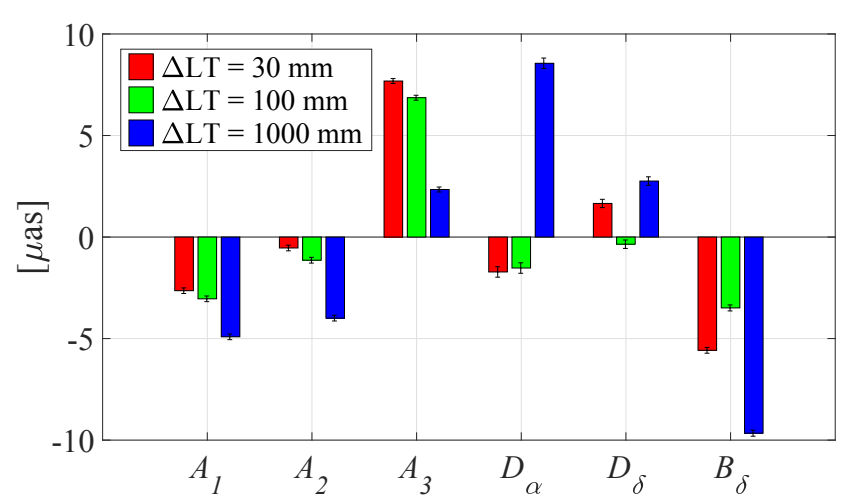

Fig. $5 \mathrm{CRF}$ transformation parameters and their standard deviations (error bars) of combined solutions with different LT selections w.r.t. VLBI-only solution.

In order to analyze the effect of the LTs on the combined solution, three types of combined solutions were computed depending on the LT threshold $\Delta \mathrm{LT}$. In the following, we search for this effect in (i) the CRF, (ii) the TRF, and (iii) the EOP.

(i) Figure 5 shows that the $A_{3}$ components of the combined solutions (A), (B), and (C) seem to get smaller when the $\Delta \mathrm{LT}$ is increased. However, they always have larger disagreement with ICRF2 compared to the VLBI-only solution (not shown). On the other hand, the other components show biggest transformation w.r.t. VLBI-only solution when $\Delta \mathrm{LT}=1000 \mathrm{~mm}$ is used. Especially, the drift of the right ascension $D_{\alpha}$ shows slightly smaller offsets in combinations using $\Delta \mathrm{LT}=30 \mathrm{~mm}$ and $\Delta \mathrm{LT}=100 \mathrm{~mm}$, but it is significantly increased if $\Delta \mathrm{LT}=1000 \mathrm{~mm}$ is used. This behavior might be explained by the fact that, in the combined solutions, $\Delta \mathrm{UT} 1$ is derived from VLBI, GNSS, and SLR data and the combined network is de- 


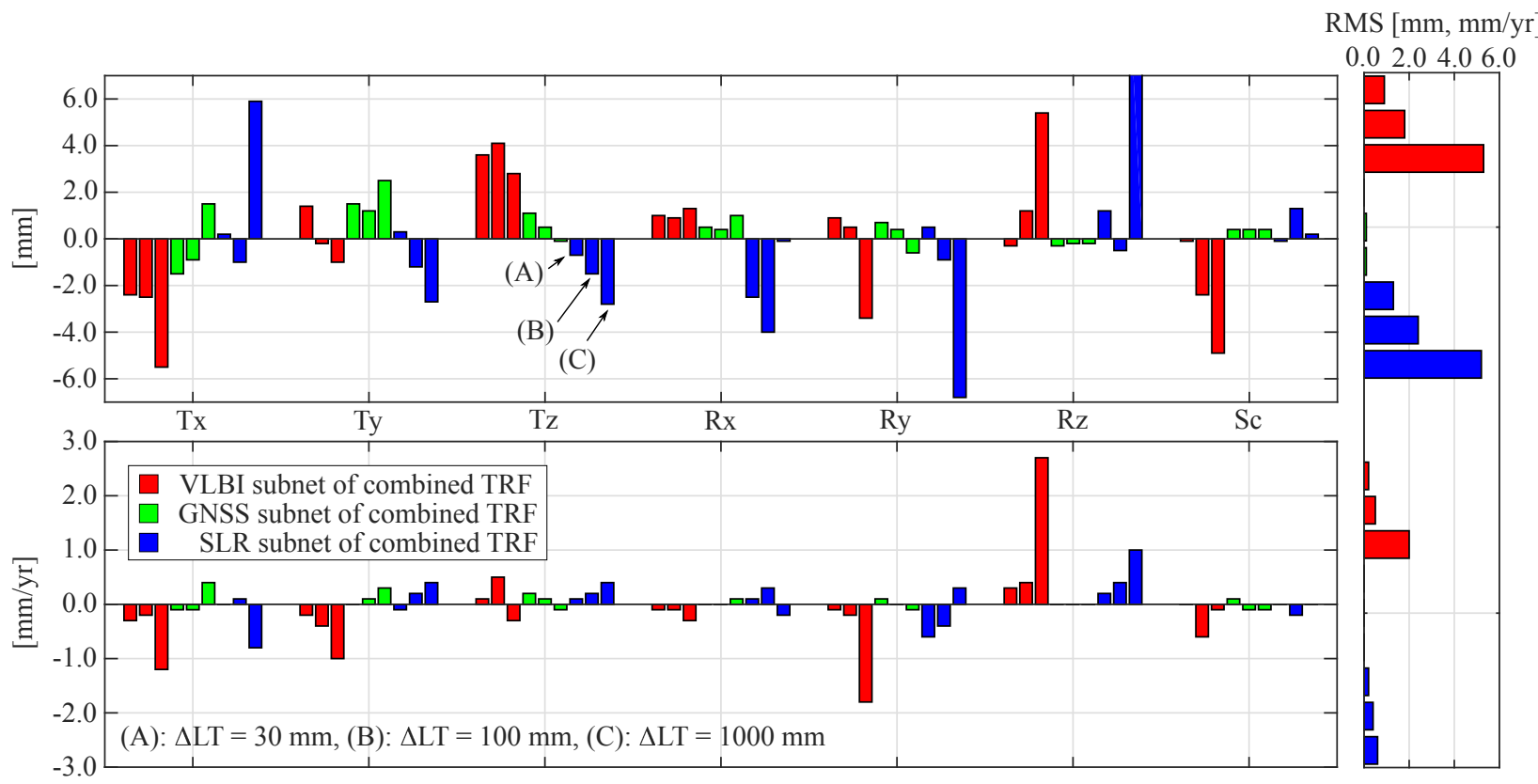

Fig. 6 Estimated offsets (upper left) and rates (lower left) of a 14-parameter TRF similarity transformation of the techniquespecific subnets of the combined TRF solution w.r.t. single-technique multi-year solutions. In addition, the right plot shows the root mean square (RMS) of the transformation residuals which indicates the grade of the deformation of each subnet due to the combination. The RMS values of GNSS are so small that they are not visible in this scale. Each subnet (VLBI: red, GNSS: green, SLR: blue) is represented by three bars per parameter which are related to the combined solution setups (A), $(\mathrm{B})$, and $(\mathrm{C})$.

formed (see Fig. 6) to a certain extent by discrepant co-locations (using the large LT threshold).

(ii) The impact of LT selection on the TRF is studied by analyzing the results of a 14-parameter TRF similarity transformation between the technique-specific subnets of the combined solutions (A), (B), and (C) and the single-technique multi-year solutions. For the transformations, the same subnet as for the datum conditions of the single-technique solutions was used. Figure 6 shows the TRF transformation parameters, their rates and the root mean squares (RMS) of the transformation residuals. Thereby, the investigation should focus on the interpretation of all parameters since (a) the TRF datum parameters of the combined solution allow an evaluation of the quality of the realized datum (origin by SLR, orientation by NNR on GNSS subnet, scale by SLR and VLBI) and (b) the interpretation of the other TRF transformation parameters allows to evaluate the ability of the selected LTs to transfer the datum information from one subnet to the other.

It is clearly visible that SLR and VLBI are sensitive on the LT selections since their global station distribution is only sparse. In the case of SLR, even though it is the unique contributor to the origin of the TRF, the translations are significantly influenced by the LT selection. When including nearly all LTs (solution C; $\Delta \mathrm{LT}=1000 \mathrm{~mm}$ ), the SLR and VLBI subnets of the combined solution are clearly translated w.r.t. the single-technique solutions. The origin of the combined solution (B) is not affected that much by the LTs compared to the SLR solution, whereas solution (A) agrees quite well with it. The same holds for the transfer of the origin information from SLR to the VLBI and GNSS subnets. Solution (C) shows the worst performance in $x$ whereas solution (B) shows the largest translation offsets and rates in $z$ of the VLBI subnet.

The orientation of the GNSS subnet is not affected that much by the combination regardless of the LT selection because the orientation of those frames was constrained to the DTRF2014 GNSS subnet by an NNR condition. In case of VLBI and SLR, it can be concluded that solution $(\mathrm{C})$ clearly rotates the subnets w.r.t. the single-technique solutions. The smallest sum of rotations is achieved by the LT selection of solution (B).

The scale parameter is the most critical issue in this combination since it absolutely relies on the quality of the scale transfer via the SLR-VLBI LTs at colocation sites. From Table 3 it is clearly visible that only a small number of LTs is selected (4), if a threshold $\Delta \mathrm{LT}=30 \mathrm{~mm}$ is used. Nevertheless, these four SLRVLBI LTs allow a scale realization which is comparable to the scale of the VLBI and SLR single-technique solutions. Furthermore, in solution (A), the intrinsic scales of the SLR and VLBI subnets in the combined solu- 
tion are nearly the same. In solution (B), the SLR and VLBI scales do not agree to each other and both show larger offsets to the technique-specific networks than solution (A). Solution (C) results in the largest scale discrepancies. For the scale transfer to GNSS, all solutions perform equal.

The mean network deformation of the combined TRF w.r.t. the single-technique solutions is shown in the right panel of Fig. 6. It seems that the GNSS subnet is rather stable which explains why the GNSS network is not affected at all by the LT selections. The VLBI and SLR networks are deformed by up to $6 \mathrm{~mm}$ and by up to $2 \mathrm{~mm} / \mathrm{yr}$ in $3-\mathrm{D}$ position and velocity vectors, respectively, depending on $\mathrm{LT}$ selections.

Besides the TRF comparison to the single-technique solutions, the combined solution was also compared to the DTRF2014. The transformation parameters show a similar behavior as discussed before which means that the combined TRF solutions (A) and (B) are reliable TRF solutions. In conclusion, we can state that the most stable and DTRF2014-like TRF datum is realized by the LT threshold $\Delta \mathrm{LT}=30 \mathrm{~mm}$ in solution (A). However, solution (B) with $\Delta \mathrm{LT}=100 \mathrm{~mm}$ also results in stable and reliable TRF datum parameters.

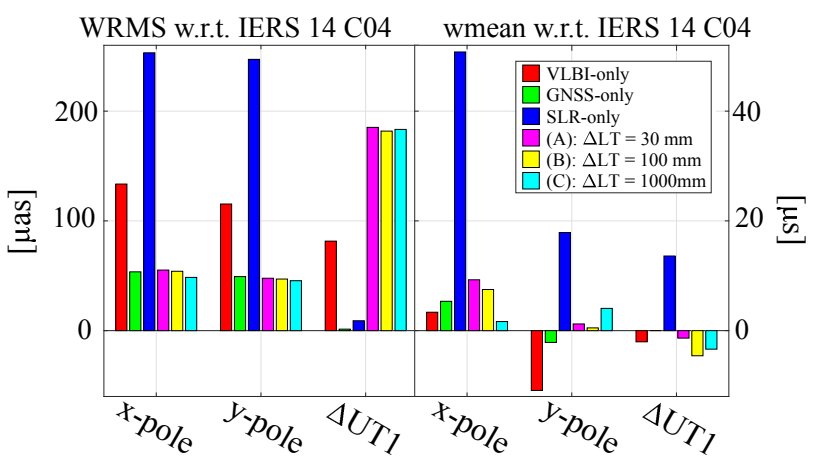

Fig. 7 EOP comparison of the single-technique multi-year solutions (VLBI-only: red, GNSS-only: green, SLR-only: blue) and the combined solutions with different LT selections $(\Delta \mathrm{LT}=30 \mathrm{~mm}:$ magenta $, \Delta \mathrm{LT}=100 \mathrm{~mm}:$ yellow,$\Delta \mathrm{LT}=$ $1000 \mathrm{~mm}$ : cyan) w.r.t. the IERS $14 \mathrm{C} 04$ time series. The left panel shows the weighted root-mean-square values (WRMS); the right panel shows the weighted mean (wmean) values. Left vertical axis denotes the scale of $\mathrm{x} / \mathrm{y}$-pole, and right one indicates that of $\Delta \mathrm{UT} 1$.

(iii) In this last subsection, the impact of the LT selection on the estimated EOP is assessed. From Fig. 7 , it can be clearly seen that GNSS is dominating, especially the determination of the terrestrial $x / y$-pole coordinates. For the WRMS values, the LT selection does not matter at all. In case of the wmean values, the pole coordinates are affected by different LT selections. However, there are no big discrepancies $(1.5 \mathrm{~mm}$ at maxi- mum at the Earth's surface) among the LT selections. The impact of different EOP combination setups will be discussed in Sect. 5.2 .

The $x / y$-pole wmean values of the SLR-only solution are significantly larger than those of the other solutions. This phenomenon is caused by a small drift since around 2010 when some SLR stations were affected by huge earthquakes. Therefore, the artificial realization of the orientation (via NNR condition) could not be realized as well as for the other techniques. However, this effect does not affect the combined solution at all since only datum-free NEQs are combined.

\subsection{Impact of EOP combination setups}

In order to evaluate the impact of the combined EOP on the estimated parameters and especially the CRF, four different EOP combination setups are computed (Table 4).

Figure 8 shows the impact of the different EOP combination scenarios on the technique-specific subnets of the combined TRF solution. Systematic effects can be found especially in the $\mathrm{Rz}$ transformation parameters of the VLBI and the SLR subnet of the combined solution w.r.t. DTRF2014. Figure8 shows a small difference between including and excluding $\triangle \mathrm{UT} 1$ due to correlations. Since the orientation of the combined station network is realized using a selected GNSS subnet, this subnet is not affected at all by the EOP combination setups. Besides, the EOP combination setups scarcely influence the estimated scale parameters.

Figure 9 shows the CRF transformation parameters of the combined solutions w.r.t. VLBI-only solution. The solution (D) (green bars) agrees within $1 \mu$ as except for a small bias of $3.4 \mu$ as. This means since no EOP are combined at all, the CRF is not affected by the combination of the station coordinates via the LTs. However, as shown in Fig. 10, the standard deviations of the source coordinates are decreased, i.e., improved, after the combination of station coordinates only. In particular, the declinations of the VCS sources and newly added sources which were not included in ICRF2 are improved (smaller standard deviations) significantly in the southern hemisphere (Fig. 10). The mean values of the improvement for those sources are about $32 \mu$ as and $57 \mu$ as for the northern and southern hemisphere, respectively. The improvements in percentages are around $9 \%$ for every source type. As the standard deviations of the non-VCS sources including defining sources are smaller than those of the VCS sources, their changes are hardly recognizable in Fig. 10. Fig. 11 depicts the standard deviations of the source coordinates for the defining sources only. It can be seen that the standard 


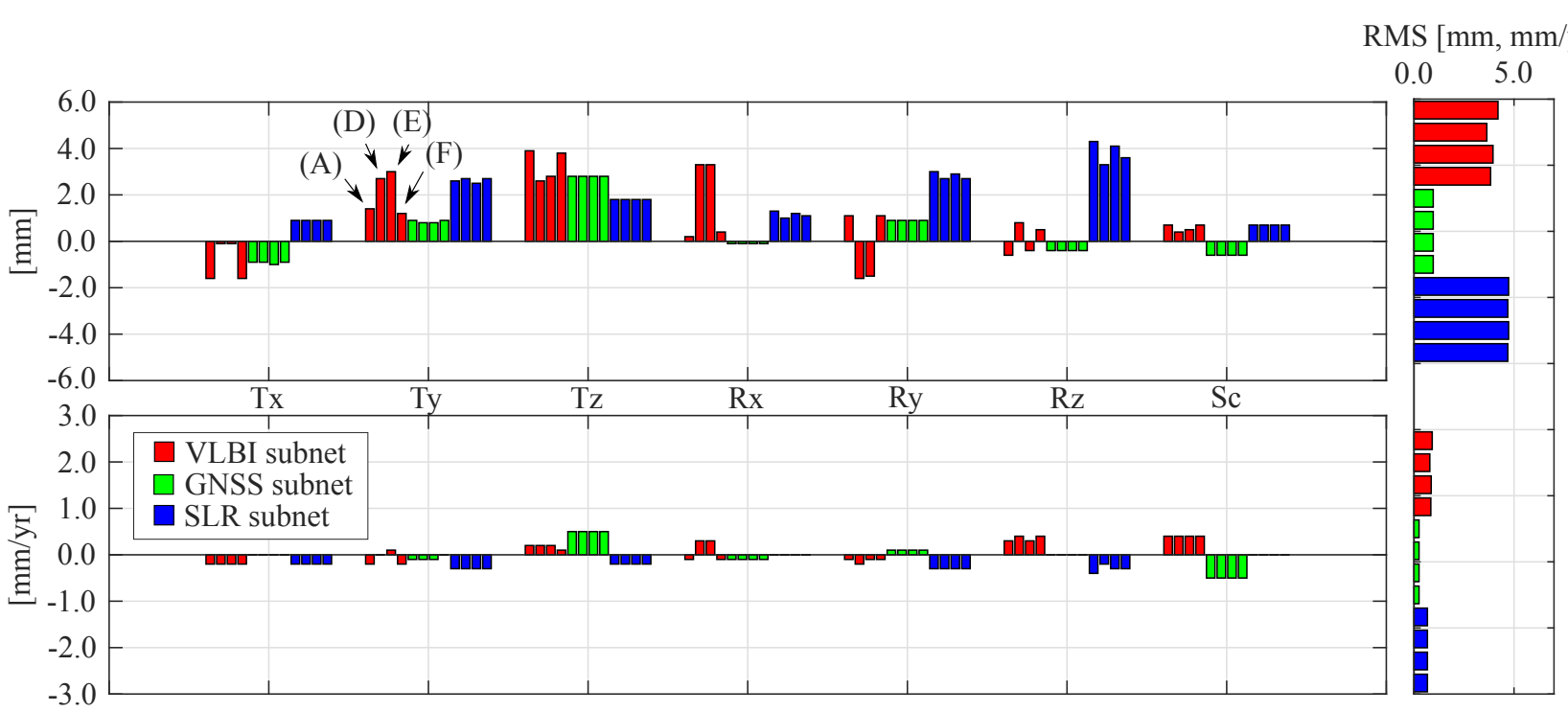

Fig. 8 Estimated offsets (upper left panel) and rates (lower left panel) of a 14-parameter TRF similarity transformation parameters of the technique-specific subnets of the combined solutions (A), (D), (E), (F) w.r.t. DTRF2014 and the mean RMS of the transformation residuals (right panel).

deviations after the combination get reduced proportional to the magnitude of the original standard deviations (VLBI-only). Thus, the southern sources (lower than $-30^{\circ}$ ) benefit the most.

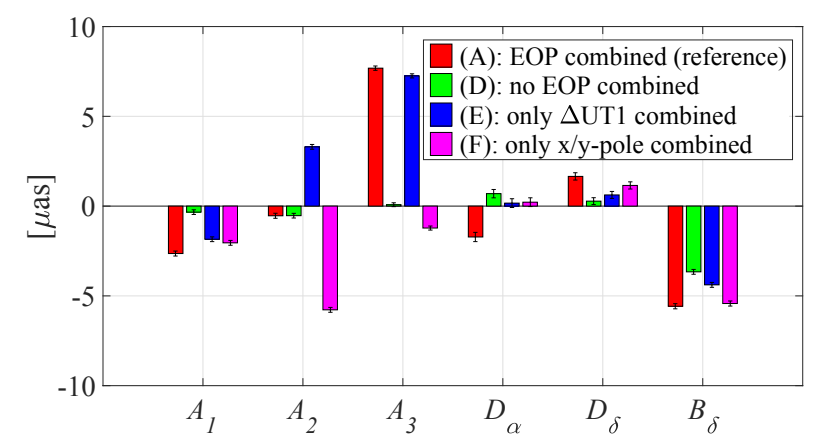

Fig. $9 \mathrm{CRF}$ transformation parameters and their standard deviations (error bars) of different EOP combination setups w.r.t. VLBI-only solution.

It has to be mentioned here that in a previous study done by Seitz et al. (2012), the VCS sources were improved by up to 4 mas which is one order of magnitude larger than this study. However, Seitz et al. (2012) only used data until 2007 while this study used data between 2005 and 2015. This means, within this study, the VCS sources were observed more often through the so-called VCS-II campaigns which were held in 2014 and 2015.

The combination of the terrestrial $x / y$-pole coordinates (F) improves the agreement with ICRF2 compared to VLBI-only solution for $A_{2}$ and $A_{3}$ components, $17 \%$ and $70 \%$, respectively (indirectly shown

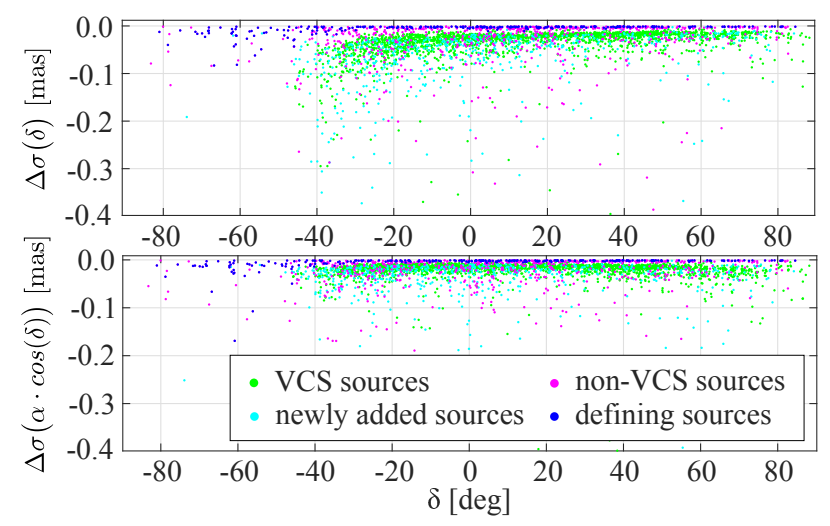

Fig. 10 Differences of source declination standard deviations $\Delta \sigma(\delta)$ and right ascension standard deviations $\Delta \sigma(\alpha \cdot \cos (\delta))$ between the combined solution (D) and the VLBI-only solution. The standard deviations of the VCS sources (green) and newly added sources (cyan), which were not included in ICRF2, are changed significantly. Negative differences mean improvement after the combination. Every combination setup so far (solutions $(A)-(F)$ ) shows a similar plot except the solution with the down-weighted VLBI NEQ (G).

in Figs. (4 9). On the other hand, there is almost no effective impact on the components $D_{\alpha}$ and $D_{\delta}$, and $A_{1}$ component disagrees more $(25 \%)$. As discussed in the beginning of Sect. 5 , the declination biases which appear in recent years due to the new Australian VLBI network are clearly detected in all combination setups and VLBI-only solution. Therefore, at this stage, it is hard to gauge which declinations are true and if the combined solution improves/degrades the declination of the CRF. Meanwhile, in the combination of the terres- 


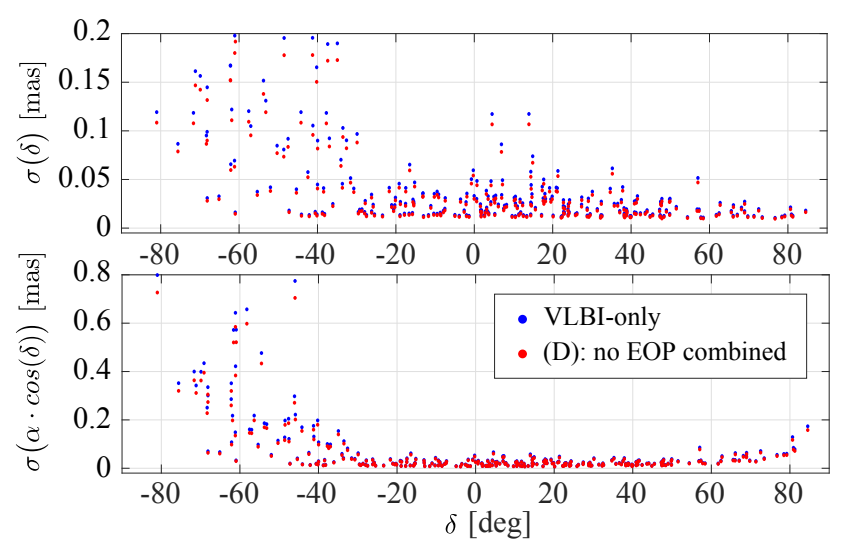

Fig. 11 The standard deviations of the declination $\sigma(\delta)$ and right ascension $\sigma(\alpha \cdot \cos (\delta))$ for defining sources in the VLBIonly and combined solution (D). Every combination setup so far (solutions $(\mathrm{A})-(\mathrm{F})$ ) shows a similar plot except the solution with the down-weighted VLBI NEQ (G). The improvements in percentage are around $9 \%$.

trial $x / y$-pole coordinates $(\mathrm{F})$, the correlated parameters, right ascension and $\Delta \mathrm{UT} 1$, get less correlated. This fact supports the improvement of $A_{3}$ components in the solution $(\mathrm{F})$.

When the $\Delta \mathrm{UT} 1$ is included in the combination, it is clearly seen that the $A_{3}$ components, which are directly related to the right ascension (Eq. 2), are mostly affected ((A) and (E) in Fig. 9). It can be easily expected that the deteriorated $\frac{d}{d t}(\Delta \mathrm{UT} 1)$ from the satellite techniques disturb the accurate determination of $\triangle \mathrm{UT} 1$ to a certain extent. However, including $\Delta \mathrm{UT} 1$ from the satellite techniques is of main importance for its densification in time. Therefore, it is desirable to improve $\Delta \mathrm{UT} 1$ accuracy of the satellite techniques before the combination and/or apply a proper constraint for $\triangle \mathrm{UT} 1$ of the satellite techniques during the combination.

\subsection{Impact of weighting}

To investigate the impact of the satellite techniques on the VLBI parameters, one combination setup was tested where the VLBI NEQ was down-weighted by a factor of 10 w.r.t. the GNSS and SLR NEQ. Figure 12 shows the CRF transformation parameters w.r.t. VLBIonly solution (only defining sources are used) of two combined solutions using different weights for the VLBI NEQ $\left(\lambda_{\mathrm{VLBI}}=1.0\right.$ and $\left.\lambda_{\mathrm{VLBI}}=0.1\right)$. When the VLBI NEQ is down-weighted, all CRF transformation parameters increase (especially $A_{3}$ to $\left.50 \mu \mathrm{as}\right)$. This means that the CRF is influenced by dominating satellite techniques in the TRF and EOP combination. However, if the TRF and the EOP benefit from this, also the CRF might be improved. When the source positions of the two combined solutions are compared to the VLBI-only $\mathrm{CRF}$, the impact of the different weights can be clearly seen (Fig. 13).

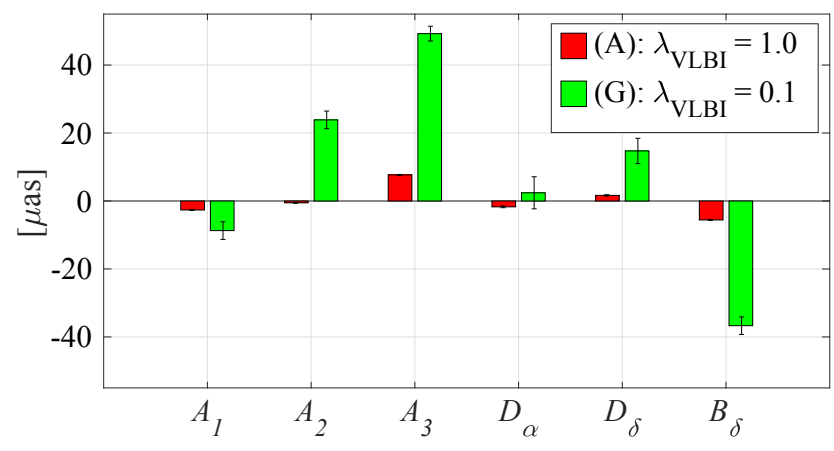

Fig. 12 CRF transformation parameters and their standard deviations (error bars) of the combined solutions with different weights for the VLBI NEQ w.r.t. VLBI-only solution.

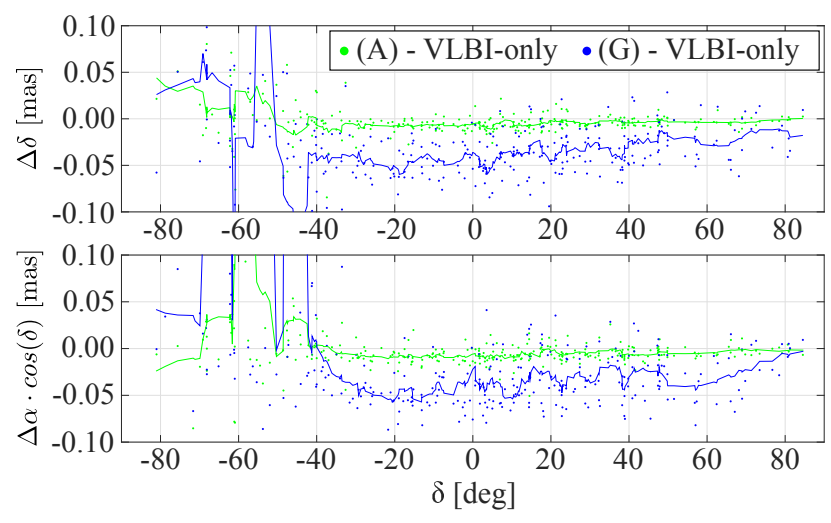

Fig. 13 Source position differences between the combined solution (A) and the VLBI-only solution (green) and the combined solution $(\mathrm{G})$ and the VLBI-only solution (blue) for the declination $\delta$ (upper panel) and the right ascension $\alpha$ (lower panel). Only defining sources are considered. The solid lines denote sliding medians.

The impact of different VLBI NEQ weights on the EOP is shown in Fig. 14. The WRMS and wmean values of the terrestrial $x / y$-pole coordinates are comparable regardless of the VLBI weighting because the contribution of GNSS to the $x / y$-pole coordinates dominates the combination. The $\Delta \mathrm{UT} 1$ values get worse when the VLBI NEQ is down-weighted since VLBI only is capable of giving the absolute information on $\Delta \mathrm{UT} 1$, while the satellite techniques can only provide $\frac{d}{d t}(\Delta \mathrm{UT} 1)$.

All the TRF transformation parameters of the GNSS and SLR subnets in the combined solution are slightly changed by up to $2 \mathrm{~mm}$ after down-weighting the VLBI NEQ (not shown). All translations and the $\mathrm{x}$ - and $\mathrm{y}-$ rotations of the VLBI subnet TRF transformation pa- 


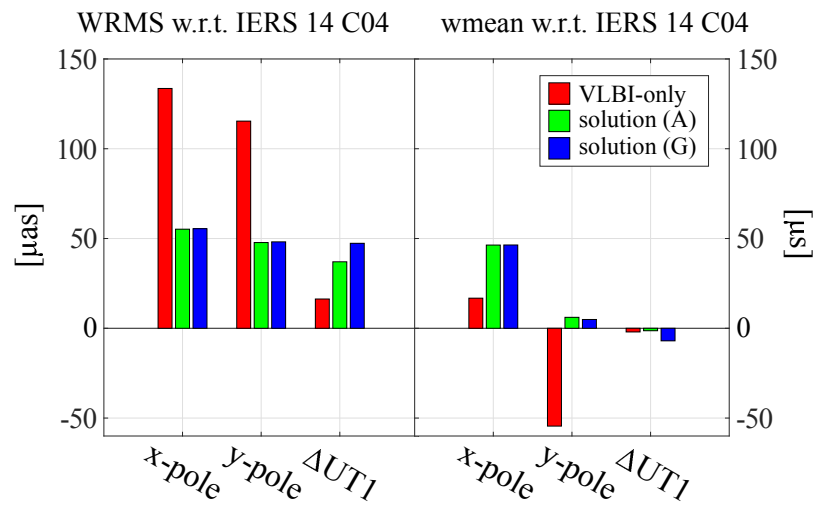

Fig. 14 EOP comparison of the VLBI-only solution (red) and the combined solutions with different weights for the VLBI NEQ $\left(\lambda_{\mathrm{VLBI}}=1.0\right.$ : green, $\lambda_{\mathrm{VLBI}}=0.1$ : blue $)$ w.r.t. the IERS 14 C04 time series. The left and right panels show the weighted root-mean-square (WRMS) values and the weighted mean (wmean) values, respectively.

rameters vary below $1 \mathrm{~mm}$. The z-rotation and the scale offsets, to which the VLBI subnet is most sensitive among the techniques, are changed significantly: -0.6 to $0.6 \mathrm{~mm}$ and 0.7 to $-0.9 \mathrm{~mm}$, respectively.

\section{Conclusions}

On the basis of 11 years (2005.0-2016.0) of GNSS, VLBI, and SLR observations, seven types of combined solutions were computed and examined for the consistency of TRF, EOP, and CRF. More precisely, the selection of the LTs by applying three different thresholds within the LT selection process was varied (solutions denoted as (A), (B), and (C)), different EOP combination setups where $\Delta \mathrm{UT} 1$ and $x / y$-pole coordinates of different techniques were combined or estimated independently (solutions denoted as (A), (D), (E), and (F)) and finally, the weight of the VLBI NEQ was varied in the combination w.r.t. the satellite techniques (solution denoted as $(\mathrm{A})$ and $(\mathrm{G})$ ). All solution setups were analyzed w.r.t. their impact on the consistently estimated parameters, and it was tested whether there exists an optimal combination setup which provides the best estimates for all parameters. Therefore, the combination results were compared to the DTRF2014 solution, the IERS 14 C04 time series, and the VLBI-only CRF solution, respectively. For all solutions, a bias in the declinations of CRF sources on the southern hemisphere was found w.r.t. ICRF2. This effect is well known in the VLBI community and could be verified in this study.

In the case of the LTs, three different LT and equal velocity thresholds were applied: $\Delta \mathrm{LT}=30 \mathrm{~mm}$ with $\Delta \mathrm{v}=1.5 \mathrm{~mm} / \mathrm{yr}(\mathrm{A}), \Delta \mathrm{LT}=100 \mathrm{~mm}$ with $\Delta \mathrm{v}=5 \mathrm{~mm} / \mathrm{yr}(\mathrm{B})$, and $\Delta \mathrm{LT}=1000 \mathrm{~mm}$ with $\Delta \mathrm{v}=$
$1000 \mathrm{~mm} / \mathrm{yr}$ (C). The combination using nearly all available LTs (C) causes a large shift of the origin, a discrepancy between the VLBI and SLR scale parameters, and a deformation of the VLBI and SLR subnets compared to DTRF2014. The solution (B) is mostly comparable to solution (A) or sometimes even better. However, for the scale offsets, a clear degradation of the consistency of the VLBI and SLR scale can be seen even for solution (B) although more LTs at co-location sites could be considered. Most of the CRF components show biggest transformation w.r.t. VLBI-only solution when $\Delta \mathrm{LT}=1000 \mathrm{~mm}$ is used. The EOP are less sensitive to different LT selections than the TRF.

Concerning the EOP, four different combinations were computed to investigate the impact of combined EOP on the TRF and the CRF solutions. The combined solution (D), where the EOP of the VLBI-only solution and those of the satellite techniques have not been combined, shows comparable results w.r.t. the VLBIonly solution. The sole combination of terrestrial $x / y$ pole coordinates $(\mathrm{F})$ is beneficial to the estimated CRF, whereas the sole combination of $\Delta \mathrm{UT} 1$ (E) causes a rotation of the CRF around the z-axis. In the TRF solutions, the largest changes can be found in the VLBI subnet, whereas the GNSS subnet is nearly not affected at all by different EOP combination setups. However, for all combination solutions, a clear decrease in the source position standard deviations can be found. The same holds for the standard deviations of the EOP itself.

The last solution tested in this investigation was the combination of the satellite technique NEQs with a down-weighted VLBI NEQ (G) to quantify the maximal impact of the satellite techniques on a consistently estimated CRF. In general, one can state that the obtained CRF is rotated w.r.t. the VLBI-only CRF by up to $40 \mu$ as since the combined EOP are totally dominated by GNSS (and SLR to a certain extent).

The results of this paper support the discussion of several aspects for the consistent estimation of TRF, EOP, and CRF compared to a separate estimation as currently performed:

- It is statistically true and could be confirmed by this study that the combination of different space geodetic techniques reduces the standard deviations of the estimated parameters due to the larger number of observations.

- The LT configuration mostly influences the TRF. Only minor impact can be seen on the CRF and the EOP.

- The estimated CRF benefits by the precise terrestrial $x / y$-pole coordinates from GNSS. Since the 
standard deviations of the pole coordinates are significantly decreased, also the effect on the CRF is assumed to be beneficial.

- The combination of $\triangle \mathrm{UT} 1$ of VLBI and the satellite techniques mainly affects the right ascension and therefore the CRF z-rotation. However, it might be beneficial that a continuous $\triangle \mathrm{UT} 1$ time series could be achieved by the combination. Nevertheless, it should be a future task to further improve the $\Delta \mathrm{UT} 1$ accuracy.

- Dominating satellite techniques significantly affect the CRF and cause systematic rotations of the source positions.

- Currently, the optimal combination setup would be $\Delta \mathrm{LT}=30 \mathrm{~mm}$ and no $\Delta \mathrm{UT} 1$ included in the combination considering the importance of scale (TRF) and frame rotation.

Since 2010, the Australian network started to join the regular IVS sessions and, since then, a bias in the declination of southern hemisphere source positions appears. As discussed in Sect. 5, there is only a minimal chance to investigate this effect in a comparison of our solutions with the ICRF2, because the ICRF includes VLBI data only until 2009 - a year before the effect occurred. Instead, our solutions should be compared with the ICRF3 which covers the period where the Australian sites contribute to the CRF. The same holds for the comparison of our results to those of Seitz et al. (2014) who considered a different observation time span and where no VCS-II sessions were included in the CRF determination. Furthermore, to avoid potential biases between the techniques, an external validation, e.g., using various source catalogs from different frequencies like Gaia (Mignard et al., 2016), should be aimed for in the future.

Finally, the IUGG resolution 3 reminds to take care of the consistency of ITRF, EOP, and ICRF during new ICRF computations despite the fact that currently different products are still computed by different institutions which creates a high hurdle for a joint estimation. For a successful consistent realization of them, the specific benefits of the simultaneous estimation should be clarified. The presented study significantly contributes to this issue but should also be extended to include all the full observation time span of all four space geodetic techniques (VLBI, GNSS, SLR, and DORIS).

Acknowledgements We thank the three anonymous reviewers for their helpful comments and suggestions. This study was funded by the German Research Foundation (DFG) within the Research Unit "Space-Time Reference Systems for Monitoring Global Change and for Precise Navigation in Space" (FOR 1503). This study made use of GNSS analysis solutions provided by the Center for Orbit Determination in Europe (CODE; Steigenberger et al., 2014).

\section{References}

Abbondanza C, Chin T, Gross R, Heflin M, Parker J, Soja B, van Dam T, Wu X (2017) JTRF2014, the JPL Kalman filter, and smoother realization of the International Terrestrial Reference System. J Geophys Res Solid Earth. doi:10.1002/2017JB014360

Altamimi Z, Sillard P, Boucher C (2002) ITRF2000: a new release of the International Terrestrial Reference Frame for earth since applications. J Geophys Res 107(B10). doi:10.1029/2001JB000561

Altamimi Z, Rebischung P, Métivier L, Collilieux X (2016) ITRF2014: A new release of the International Terrestrial Reference Frame modeling nonlinear station motions. J Geophys Res Solid Earth 124:61096131. doi:10.1002/2016JB013098

Angermann D, Drewes H, Krügel M, Meisel B, Gerstl M, Kelm R, Müller H, Seemüller W, Tesmer V (2004) ITRS combination center at DGFI a Terrestrial Reference Frame Realization 2003. Deutsche Geodätische Kommission, Reihe B, München

Angermann D, Seitz M, Drewes H (2013) Global Terrestrial Reference Systems and their realizations. In: $\mathrm{Xu}$ G(ed.) Sciences of geodesy II - innovations and future developments, Springer, Berlin, pp 97-132. doi:10.1007/978-3-642-28000-9_3

Arias EF, Charlot P, Feissel M, Lestrade J-F (1995) The extragalactic reference system of the International Earth Rotation Service, ICRS. Astron Astrophys 303: 604-608

Beasley AJ, Gordon D, Peck AB, Petrov L, MacMillan DS, Formalont EB, Ma C (2002) The VLBA calibrator survey - VCS1. Astrophys J Suppl Ser 141(1): 13-21. doi:10.1086/339806

Bizouard C, Lambert S, Becker O, Richard J (2017) Combined solution C04 for Earth rotation parameters consistent with International Terrestrial Reference Frame 2014. http://hpiers.obspm.fr/iers/eop/eopc04/C04.guide.pdf. Accessed 06 Nov 2017

Bloßfeld M. (2015) The key role of Satellite Laser Ranging towards the integrated estimation of geometry, rotation and gravitational field of the Earth. PhD thesis, Deutsche Geodätische Kommission (DGK) Reihe C, No. 745, Verlag der Bayerischen Akademie der Wissenschaften, ISBN: 978-3-7696-5157-7

Bloßfeld M, Angermann D, Seitz M (2017) DGFI-TUM analysis and scale investigations of the latest Terrestrial Reference Frame Realizations. In: IAG proceedings (submitted) 
Böckmann S, Artz T, Nothnagel A (2010) VLBI Terrestrial Reference Frame contributions to ITRF2008. J Geod 84(3): 201-219. doi:10.1007/s00190-009-03577

Dach R, Lutz S, Walser P, Fridez P (2015) Bernese GNSS software, version 5.2. Astronomical Institute, University of Bern. doi:10.7892/boris. 72297

Dick WR, Thaller D (2015) IERS annual report 2014. Verlag des Bundesamts für Kartographie und Geodäsie, Frankfurt am Main

Dow JM, Neilan RE, Rizos C (2009) The International GNSS Service in a changing landscape of global navigation satellite systems. J Geod 83(3-4): 191-198. doi:10.1007/s00190-008-0300-3

Fey AL, Gordon D, Jacobs CS (eds) (2009) The second realization of the International Celestial Reference Frame by very long baseline interferometry. IERS Technical Note, No. 35. Verlag des Bundesamtes fr Kartographie und Geodsie, Frankfurt am Main, http://www.iers.org/TN35/. ISBN: 978-389888-918-6

Fey AL, Gordon D, Jacobs CS, Ma C, Gaume RA, Arias EF, Bianco G, Boboltz DA, Böckmann S, Bolotin S, Charlot P, Collioud A, Engelhardt G, Gipson J, Gontier A-M, Heinkelmann R, Kurdubov S, Lambert S, Lytvyn S, MacMillan DS, Malkin Z, Nothnagel A, Ojha R, Skurikhina E, Sokolova J, Souchay J, Sovers OJ, Tesmer V, Titov O, Wang G, Zharov $\mathrm{V}$ (2015) The second realization of the International Celestial Reference Frame by very long baseline interferometry. Astron J 150(2): 58. doi:10.1088/0004$6256 / 150 / 2 / 58$

Gerstl M (1997) Parameterschätzung in DOGS-OC. Deutsches Geodätisches Forschungsinstitut, MG/01/ 1996/DGFI

Gerstl M, Kelm R, Müller H, Ehrnsperger W (2000) DOGS-CS: Kombination und Lösung großer Gleichungssysteme. Interner Bericht Nr. MG/01/1995/ DGFI, Deutsches Geodätisches Forschungsinstitut, Munich

Gordon D (2014) Revisiting the VLBA calibrator surveys for ICRF3. In: Behrend D, Baver KD, Armstrong KL (eds) Proceedings IVS 2014 general meeting, Science Press, Beijing, pp 386-389

Gordon D, Jacobs C, Beasley A, Peck A, Gaume R, Charlot P, Fey A, Ma C, Titov O, Boboltz D (2016) Second epoch VLBA calibrator survey observations: VCS-II. Astron J 151(6): 154. doi:10.3847/0004$6256 / 151 / 6 / 154$

IUGG (2011) Resolutions adopted by the Council at the XXV IUGG General Assembly, Melbourne, Australia. http://www.iugg.org/resolutions. Accessed 4 May 2016
Lovell JEJ, McCallum JN, Reid PB, McCulloch PM, Baynes BE, Dickey JM, Shabala SS, Watson CS, Titov O, Ruddick R, Twilley R, Reynolds C, Tingay SJ, Shield P, Adada R, Ellingsen SP, Morgan JS, Bignall HE (2013) The AuScope geodetic VLBI array. J Geod 87(6): 527-538. doi:10.1007/s00190-0130626-3

Malkin Z, Jacobs CS, Arias F, Boboltz D, Boehm J, Bolotin S, Bourda G, Charlot P, de Witt A, Fey A, Gaume R, Gordon D, Heinkelmann R, Lambert S, Ma C, Nothnagel A, Seitz M, Skurikhina E, Souchay J, Titov O (2015) The ICRF-3: status, plans, and progress on the next generation International Celestial Reference Frame. In: Malkin Z, Capitaine N (eds) Proc Journées 2014 "Systèmes de référence spatiotemporels", Pulkovo Observatory, Russia, pp 3-8

Mignard F, Klioner S, Lindegren L, Bastian U, Bombrun A, Hernndez J, Hobbs D, Lammers U, Michalik D, Ramos-Lerate M, Biermann M, Butkevich A, Comoretto G, Joliet E, Holl B, Hutton A, Parsons P, Steidelmller H, Andrei A, Bourda G, Charlot P (2016) Gaia Data Release 1: reference frame and optical properties of ICRF sources, Astron Astrophys, 595: A5. doi:10.1051/0004-6361/201629534

Pearlman MR, Degnan JJ, Bosworth JM (2002) The International Laser Ranging Service. Adv Space Res 30(2): 135-143. doi:10.1016/S0273-1177(02)00277-6

Petrov L, Kovalev YY, Fomalont EB, Gordon D (2008) The sixth VLBA calibrator survey: VCS6. Astron J 136(2): 580-585. doi:10.1088/0004-6256/136/2/580

Petrov L, Gordon D, Gipson J, MacMillan D, Ma C, Fomalont E, Walker RC, Carabajal C (2009) Precise geodesy with the very long baseline array. J Geod 83(9):859-876. doi:10.1007/s00190-009-0304-7

Rothacher M, Beutler G, Bosch W, Donnellan A, Gross R, Hinderer J, Ma C, Pearlman M, Plag HP, Richter B, Ries J, Schuh H, Seitz F, Shum CK, Smith D, Thomas M, Velacognia E, Wahr J, Willis P, Woodworth P (2009) The future global geodetic observing system (GGOS). In: Plag HP, Pearlman M (eds) Global geodetic observing system. Springer, Berlin, Heidelberg. doi:10.1007/978-3-642-02687-4_9

Schuh H, Behrend D (2012) VLBI: a fascinating technique for geodesy and astrometry. J Geodyn 61: 6880. doi:10.1016/j.jog.2012.07.007

Seitz M, Angermann D, Bloßfeld M, Drewes H, Gerstl M (2012) The 2008 DGFI realization of the ITRS: DTRF2008. J Geod 86(12): 1097-1123. doi:10.1007/ s00190-012-0567-2

Seitz M, Steigenberger P, Artz T (2012) Consistent realization of ITRS and ICRS. In: Behrend D, Baver KD (eds) Proceedings of the IVS 2012 general meeting, NASA/CP-2012-217504, pp 314-318 
Seitz M, Steigenberger P, Artz T (2014) Consistent adjustment of combined Terrestrial and Celestial Reference Frames. In: Rizos C, Willis P (eds) Earth on the edge: science for a sustainable planet. IAG symposia, vol 139. Springer, Berlin, pp 215-221. doi:10.1007/978-3-642-37222-3_28

Seitz M, Bloßfeld M, Angermann D, Schmid R, Gerstl M, Seitz F (2016) The new DGFITUM realization of the ITRS: DTRF2014 (data). Deutsches Geodätisches Forschungsinstitut, Munich. doi:10.1594/PANGAEA.864046

Steigenberger P, Lutz S, Dach R, Schaer S, Jäggi A (2014) CODE repro2 product series for the IGS. Astronomical Institute, University of Bern. doi:10.7892/boris. 75680

Titov O, Tesmer V, Boehm J (2004) OCCAM v.6.0 software for VLBI data analysis. In: Vandenberg NR, Baver KD (eds) Proceedings of the IVS 2004 general meeting, NASA/CP-2004-212255, pp 267-271

Willis P, Fagard H, Ferrage P, Lemoine FG, Noll CE, Noomen R, Otten M, Ries JC, Rothacher M, Soudarin L, Tavernier G, Valette JJ (2010) The International DORIS Service (IDS): Toward maturity. Adv Space Res, 45(12):1408-1420, doi: 10.1016/j.asr.2009.11.018

Wu X, Abbondanza C, Altamimi Z, Chin TM, Collilieux X, Gross RS, Heflin MB, Jiang Y, Parker JW (2015) KALREFA Kalman filter and time series approach to the International Terrestrial Reference Frame realization. J Geophys Res Solid Earth 120:37753802. doi: 10.1002/2014JB011622 